\title{
Influência do tempo de abertura da embalagem a vácuo na cor da carne bubalina e ovina
}

\author{
Influence of vacuum packaged opening time on \\ the color of buffalo and sheep meat
}

Lucas Cabarite de Oliveira [a], Cristiana Andrighetto [a], Patrícia Aparecida Cardoso da Luz ${ }^{[b] *}$, Sirlei Aparecida Maestá [a], Érico Rodrigues ${ }^{[c]}$, André Mendes Jorge ${ }^{[b]}$, Caroline de Lima Francisco ${ }^{[b]}$, Helena Sampaio Aranha ${ }^{[a]}$, Gustavo Antunes Trivelin ${ }^{[a]}$, Juliana Mara de Freitas Santos ${ }^{[a]}$

\footnotetext{
[a] Faculdade de Ciências Agrárias e Tecnológicas, Universidade Estadual Paulista “Júlio de Mesquita Filho” (UNESP), Dracena, SP, Brasil

${ }^{[b]}$ Faculdade de Medicina Veterinária e Zootecnia, UNESP, Botucatu, SP, Brasil

[c] Cursos de Engenharia Agronômica e Engenharia de Pesca, UNESP, Registro, SP, Brasil
}

*Autora correspondente

E-mail: patriciacardosoluz@gmail.com

\section{Resumo}

O objetivo deste trabalho foi avaliar a influência do período de abertura da embalagem a vácuo na cor da carne maturada e não maturada de bubalinos e ovinos. Foram utilizadas dez amostras de carne bubalina (músculo longissimus thoracis entre a 9a e 10ª vértebra torácica) e oito amostras de carne ovina (músculo biceps femoris), separadas em amostras apenas embaladas a vácuo e não maturadas, e amostras maturadas por 14 dias $(0$ a $\left.1^{\circ} \mathrm{C}\right)$. Foi avaliada a cor da carne (luminosidade, teor de vermelho, teor de amarelo e percepção subjetiva da diferença total de cor - $\Delta \mathrm{E}$ ) aos $0,20,40,60,80$ e 100 minutos após a abertura da embalagem. 0 delineamento experimental foi inteiramente ao acaso em esquema fatorial ( $2 \times 6 \times 2)$ com duas espécies (bubalinos e ovinos) e seis tempos de abertura da embalagem $(0,20,40,60,80$ e 100 minutos), avaliadas em dois períodos de maturação (0 e 14 dias). Observou-se efeito linear dos tempos de abertura da embalagem para teor de vermelho e quadrático para o teor de amarelo $(\mathrm{P}<0,05)$ da carne bubalina não maturada e maturada. Para a carne ovina houve efeito quadrático para teor de amarelo da carne não maturada e teor de vermelho e amarelo da carne maturada $(P<0,05)$, sendo possível determinar entre 67 e 69 minutos estabilidade na cor da carne dessa espécie. Em relação ao $\Delta \mathrm{E}$, observou-se que para a carne bubalina as amostras não maturadas e maturadas por 14 dias apresentaram variações claras ao olho humano, sem estabilidade da cor até o último tempo avaliado (100 minutos) $(\mathrm{P}<0,05)$. Em contraposição, a carne ovina não apresentou diferença total de cor significativa entre os tempos de abertura da embalagem a vácuo $(\mathrm{P}>0,05)$. Embora as mudanças tenham se apresentado evidentes ao olho humano, a ausência de diferença estatística entre os valores obtidos reforça a estabilidade da cor da carne dessa espécie. Conclui-se que mesmo com 100 minutos de abertura da embalagem a vácuo, a carne bubalina ainda apresenta alterações na cor, sendo necessário rever para essa espécie a recomendação do tempo 
de abertura de 30 minutos da embalagem a vácuo. Para a carne ovina a estabilização da cor ocorre aos 67 e 69 minutos após a abertura da embalagem.

Palavras-chave: Biceps femoris. Embalagem a vácuo. Intensidade de vermelho. Longissimus thoracis. Luminosidade.

\section{Abstract}

The objective of this study was to evaluate the influence opening period of vacuum packaging in the color aged meat, and not aged of buffaloes and sheep. Ten samples of buffalo meat (longissimus thoracis muscle between the 9 th and 10th thoracic vertebrae) and eight samples of sheep meat (biceps femoris muscle) were used, divided into: only wrapped samples vacuum and not aged, and samples aged for 14 days $\left(0\right.$ to $\left.1^{\circ} \mathrm{C}\right)$. Meat color was evaluated (lightness, redness, yellowness and subjective perception of color difference $(\Delta E)$ ), at 0, 20, 40, 60, 80 and 100 minutes after package opening. The experimental design was completely randomized in a factorial scheme (2x $6 \times 2$ 2) with two species (buffaloes and sheep) and six packaging opening times (0, 20, 40, 60, 80 and 100 minutes), evaluated in two ageing periods ( 0 to 14 days). Linear effect was observed of package opening time to red color and quadratic effect to the yellow content $(P<0.05)$, for not aged and matured meat. For the sheep meat a quadratic effect was found for yellow color for meat not matured and red and yellow color for aged meat $(P<0.05)$. It was possible to determine the stability of color sheep meat between 67 and 69 minutes. In relation to the $\Delta E$, it was observed that for buffalo meat, samples not aged and aged for 14 days presented very evident and clear variations to the human eye, without stability of the color until the last evaluated time (100 minutes) ( $P<0.05)$. In contrast, sheep meat did not present total differences between the opening times of the vacuum package $(P>0.05)$. In addition, although the changes have been evident to the human eye, the lack of statistical difference reinforces the color stability of the meat from this species. We concluded that even with 100 minutes of vacuum opening packaging, in buffalo meat still occurs changes in color, thus, it is necessary to review the recommendation of 30 minutes of vacuum packaging opening time. For sheep meat, color stabilization occurs between 67 and 69 minutes after package opening.

Keywords: Biceps femoris. Vacuum packaging. Redness. Longissimus thoracis. Lightness.

\section{Introdução}

O Brasil figura como primeiro produtor de carne bubalina do Ocidente, possuindo atualmente um rebanho de aproximadamente 1.150 .000 cabeças de bubalinos (MAPA, 2016).

Em relação aos ovinos, o Brasil concentra o 18o maior rebanho do mundo, apresentando a tendência de crescimento do efetivo nos últimos 10 anos (Faostat, 2015). Talvez a menor concentração regional dos animais e a crescente demanda por produtos da ovinocultura nos últimos anos sejam algumas das razões que dão suporte ao crescimento do rebanho no país (Magalhães et al., 2016). Apesar desse cenário de crescimento, o Brasil ainda importa carne ovina para abastecer o mercado consumidor interno, visto que a oferta é inferior à demanda (Viana, 2008).

A carne ovina é fonte de proteína semelhante às de outras espécies (Barreto Neto, 2010). Assim como a carne bubalina, possui baixo teor de colesterol e ácidos graxos saturados (quando comparada à carne bovina) e excelentes atributos de palatabilidade (maciez, sabor e suculência) (Giordano et al., 2010, Giuffrida-Mendoza et al., 2015; Huerta-Leidenz et al., 2016; Mello et al., 2017), conquistando assim, adeptos nos grandes centros urbanos brasileiros.

Com isso, para atender à demanda desse nicho de 
mercadoénecessário o incentivo ao uso de tecnologias que melhorem a qualidade da carne dessas espécies, como o emprego de processos de amaciamento da carne, dentre os quais destaca-se a maturação. A maturação permite que mudanças significativas na estrutura miofibrilar promovam o amaciamento e alterem as características de qualidade da carne (Palka, 2003), tornando-a também mais aromática (Pardi et al., 1995) e saborosa (Lawrie, 1985).

A maturação inicia-se após a resolução do rigor mortis, com a ruptura da linha $\mathrm{Z}$ e de outras proteínas do citoesqueleto. No entanto, é em determinados períodos de estocagem (entre 10 a 21 dias) e em ambiente refrigerado (entre $0^{\circ} \mathrm{C}$ e $1^{\circ} \mathrm{C}$ ), que ocorre o prolongamento das atividades das enzimas endógenas (calpaínas e catepsinas) (Penny, 1984). Estas enzimas são responsáveis pela degradação das proteínas miofibrilares, melhorando consideravelmente a maciez da carne (Andrighetto et al., 2006).

Durante esse processo, torna-se então necessário embalar a carne a vácuo, para retardar o crescimento de bactérias aeróbicas putrefativas e favorecer o crescimento de bactérias láticas, que podem produzir compostos antimicrobianos (Puga et al., 1999). Entretanto, quando a carne é embalada a vácuo, a cor pode se tornar escura e não atrativa para o consumidor no momento da compra. Esse é um fenômeno natural, no qual a mioglobina, proteína sarcoplasmática da fibra muscular, em ausência de oxigênio assume a coloração vermelho escuro. Porém, quando exposta ao oxigênio, após a abertura da embalagem, a ligação com esse elemento traz de volta sua coloração vermelho brilhante (Abularach et al., 1998).

Para que a carne embalada a vácuo retorne à coloração vermelha brilhante é necessário um período de exposição ao oxigênio atmosférico de aproximadamente 30 minutos após a abertura da embalagem, para permitir a oxigenação superficial da mioglobina. Esse período de tempo é descrito na literatura para a oxigenação de carne bovina (Abularach et al., 1998; Santos 2015; Barducci et al., 2016), e embora seja também utilizado para a carne de outras espécies, como a carne bubalina e ovina, são necessários mais estudos para determinar o tempo necessário para a oxigenação da mioglobina da carne dessas espécies.
Dessa forma, em virtude da cor da carne ser um dos atributos mais importantes para a decisão de compra dos consumidores, é importante conhecer o período de tempo de exposição necessário para o desenvolvimento de cor vermelha brilhante, após a abertura da embalagem, em carne maturada ou não estocada a vácuo de diferentes espécies. Portanto, o objetivo deste trabalho foi avaliar a influência do tempo de abertura da embalagem a vácuo sobre a cor da carne maturada e não maturada de bubalinos (Bubalus bubalis) e ovinos, ambos de descarte, propondo esclarecimentos para os consumidores e avanços para a pesquisa da carne dessas espécies.

\section{Material e métodos}

O experimento foi realizado na Faculdade de Ciências Agrárias e Tecnológicas - UNESP, Campus de Dracena - SP. Foram utilizadas duas espécies animais, compostas por dez novilhas bubalinas da Raça Murrah com 32 a 36 meses de idade, e oito ovelhas de descarte sem raça definida com mais de cinco anos de idade, provenientes de rebanhos da região de Dracena ( $21^{\circ} 29^{\prime} 00^{\prime \prime S}, 51^{\circ} 32^{\prime} 1$ "W, 419 m altitude).

As búfalas e as ovelhas foram abatidas em frigoríficos comerciais, localizado na cidade de Bariri-SP (370 km de Dracena) e Lupércio-SP (230 km de Dracena), respectivamente. As meias-carcaças permaneceram na câmara frigorífica $\left(0 \pm 2^{\circ} \mathrm{C}\right)$ durante 24 horas para o estabelecimento de rigor mortis. Após 24 horas na câmara de resfriamento, as amostras de carne bubalina (músculo longissimus thoracis, removido entre a 9a e 10a vértebra torácica) e de carne ovina (músculo biceps femoris) foram colhidas e posteriormente transportadas em caixa térmica para o Laboratório de Bromatologia da Faculdade de Zootecnia da UNESP, Campus Dracena.

Das amostras foram separadas as subamostras em cortes transversais com aproximadamente $2,54 \mathrm{~cm}$ de espessura e peso médio de 98,44 \pm 43,88 g. Os cortes foram realizados em serra fita (modelo 255, Beccaro, ON, Canada). Em seguida, as subamostras foram embaladas a vácuo (embalagem com 0,12 cm de espessura e com barreira ao oxigênio) e separadas para congelamento direto (não-maturadas) ou maturadas por 14 dias em câmara de maturação B.O.D. (TE-371, Tecnal, SP, 
Brasil), com temperatura de $0 \pm 1^{\circ} \mathrm{C}$ em ausência de luz, até o momento das análises de cor.

Para as análises, as subamostras não-maturadas foram descongeladas em câmara de maturação $\left(2 \pm 3^{\circ} \mathrm{C}\right)$ por $12 \mathrm{~h}$. Em seguida, as embalagens foram abertas e a cor da carne das duas espécies foi avaliada a 0, 20, 40,60 e 100 minutos após a abertura da embalagem. Foi realizado o mesmo procedimento para as subamostras maturadas, no entanto, sem a necessidade de descongelamento em função da temperatura de maturação não proporcionar congelamento das subamostras.

A cor da carne foi determinada mediante leitura em três pontos aleatórios da superfície de corte do músculo longissimus thoracis e biceps femoris de cada subamostra não maturada e maturada por 14 dias, por meio de espectrofotômetro portátil (CR-410-Konica Minolta, Câmera Co., Ltda Osaka, Japão) com iluminante D65, abertura de $8 \mathrm{~mm}$ de diâmetro e ângulo de observação de 10을 (AMSA, 2012), previamente calibrado com padrão branco, conforme instruções do fabricante. Foi considerado o sistema CIELAB por meio de leituras de refletância da luz em três dimensões: L* (luminosidade), a* (vermelho) e $b^{*}$ (amarelo), segundo metodologia descrita por Honikel (1998). A análise foi realizada no Laboratório de Bromatologia da UNESP/Dracena.

As determinações das variações globais da cor $(\Delta E)$ foram feitas de acordo com MacDougal (1994) usando as coordenadas de luminosidade $\left(\mathrm{L}^{*}\right)$, teor de vermelho $\left(a^{*}\right)$ e intensidade de amarelo $\left(b^{*}\right)$, obtidas nas determinações colorimétricas com as seguintes fórmulas: $\Delta \mathrm{E}=\left(\Delta \mathrm{L}^{2}+\Delta \mathrm{a}^{2}+\Delta \mathrm{b}^{2}\right)^{0,5}$.

Todo experimento foi realizado de acordo com os princípios éticos na experimentação animal (protocolo $n^{-}$25/2014) determinados pela Comissão de Ética em Uso de Animais (CEUA) da referida instituição.

\section{Análise estatística}

0 delineamento experimental foi inteiramente ao acaso em fatorial $(2 \times 6 \times 2)$ com duas espécies (bubalinos e ovinos) e seis tempos de abertura da embalagem $(0,20,40,60,80,100$ minutos $)$, avaliadas em dois períodos de maturação (0 e 14 dias).
Os dados foram submetidos ao teste de ShapiroWilk para verificar a suposição de normalidade ao nível de significância de 5\%. Uma vez que os dados se apresentaram normais, realizou-se análise de regressão polinomial para cada uma das variáveis de cor, ao nível de significância de 5\%. 0 Teste Tukey fui utilizado para avaliar as variações globais da cor $(\Delta \mathrm{E})$ nos diferentes tratamentos, sendo as médias consideradas significativamente diferentes quando $\mathrm{P}<0,05$.

\section{Resultados e discussão}

\section{Carne bubalina}

A luminosidade $\left(\mathrm{L}^{*}\right)$, intensidade de vermelho $\left(a^{*}\right)$ e intensidade de amarelo (b*) em função dos tempos analisados após a abertura da embalagem a vácuo da carne bubalina (Tabela 1) apresentaram comportamento semelhante em carnes não maturadas e maturadas por 14 dias; ou seja, em ambas a $L^{*}$ não foi afetada pelos diferentes tempos $(\mathrm{P}>0,05), \mathrm{a}^{*}$ apresentou regressão linear crescente $(\mathrm{P}<0,05)$ e $\mathrm{b}^{*}$ obteve comportamento quadrático $(\mathrm{P}<0,05)$.

Para a intensidade de vermelho houve efeito linear crescente $(P<0,05)$, demonstrando que a atividade da mioglobina em contato com o oxigênio não se estabilizou mesmo passados 100 minutos. 0 mesmo foi observado na intensidade de amarelo, que apresentou aumento nos valores médios ao longo dos diferentes tempos avaliados $(\mathrm{P}<0,05)$, não apresentando estabilidade para essa coordenada de cor. Portanto, até 100 minutos da abertura da embalagem houve alteração na cor da carne.

Quanto aos valores das variações globais da cor $(\Delta E)$ - em escala sugerida por Prändl et al. (1994), com a seguinte classificação: 0-0,2 correspondem a alterações imperceptíveis ao olho humano; 0,20,5 , muito pouco perceptíveis; $0,5-1,5$, pouco perceptíveis; 1,5-3,0, percepções evidentes; 3,06,0, percepções muito evidentes; 6-12, percepção bastante clara; 12-14, facilmente perceptíveis - é possível verificar que a variação da cor da carne bubalina do presente estudo apresentou-se evidente e perceptível ao olho humano a partir do primeiro tempo de avaliação (Figura 1). 
Tabela 1 - Cor instrumental do músculo longissimus thoracis de bubalinos não maturado (0 dias de maturação) e maturado (14 dias de maturação), avaliado em diferentes tempos após ser retirado da embalagem a vácuo

0 dias de maturação

\begin{tabular}{|c|c|c|c|c|c|c|c|c|}
\hline \multirow{2}{*}{ Itens } & \multicolumn{6}{|c|}{ Tempo de abertura da embalagem (min) } & \multirow{2}{*}{ Cv (\%) } & \multirow{2}{*}{$\mathbf{R}$} \\
\hline & 0 & 20 & 40 & 60 & 80 & 100 & & \\
\hline $\mathrm{L}^{*}$ & 33,65 & 32,77 & 33,81 & 33,69 & 33,28 & 33,59 & 3,74 & Ns \\
\hline$a^{*}$ & 16,49 & 18,84 & 19,30 & 19,70 & 19,32 & 22,38 & 8,03 & $L^{1}$ \\
\hline $\mathbf{b}^{*}$ & 5,10 & 8,53 & 9,01 & 9,26 & 9,15 & 11,49 & 9,85 & $Q^{2}$ \\
\hline \multicolumn{9}{|c|}{14 dias de maturação } \\
\hline \multirow{2}{*}{ Itens } & \multicolumn{6}{|c|}{ Tempo de abertura da embalagem (min) } & \multirow{2}{*}{ CV (\%) } & \multirow{2}{*}{$\mathbf{R}$} \\
\hline & 0 & 20 & 40 & 60 & 80 & 100 & & \\
\hline $\mathrm{L}^{*}$ & 35,05 & 32,46 & 35,08 & 34,61 & 34,57 & 33,80 & 5,12 & Ns \\
\hline$a^{*}$ & 15,85 & 19,43 & 20,78 & 21,53 & 21,61 & 22,38 & 14,42 & $L^{1}$ \\
\hline $\mathbf{b}^{*}$ & 6,13 & 9,76 & 10,47 & 11,04 & 11,17 & 11,42 & 11,55 & $\underline{Q}^{2}$ \\
\hline
\end{tabular}

Nota: $L^{*}=$ Luminosidade; $a^{*}=$ Intensidade de vermelho; $b^{*}=$ Intensidade de amarelo.

$C V=$ Coeficiente de variação. $R=$ Regressão. Ns $=$ Não significativo. $L=$ Regressão Linear. $Q=$ Regressão Quadrática.

0 dias de maturação: $1 Y=0,0447 x+17,102\left(R^{2}=0,79\right) ; 2 Y=-0,0003 \times 2+0,0834 x+5,8617\left(R^{2}=0,81\right)$.

14 dias de maturação: $1 Y=0,0571 x+17,408\left(R^{2}=0,80\right) ; 2 Y=-0,0009 x 2+0,1303 x+6,6256\left(R^{2}=0,93\right)$.

0 dias de maturação

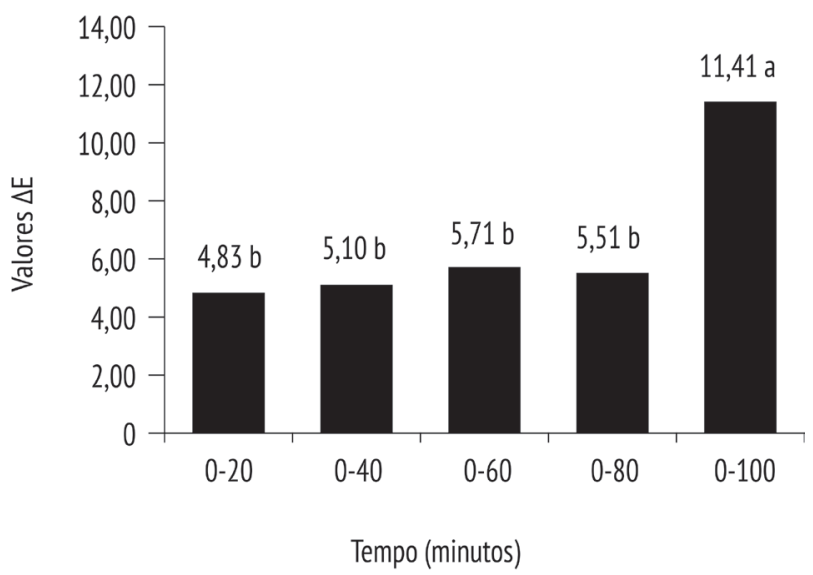

14 dias de maturação

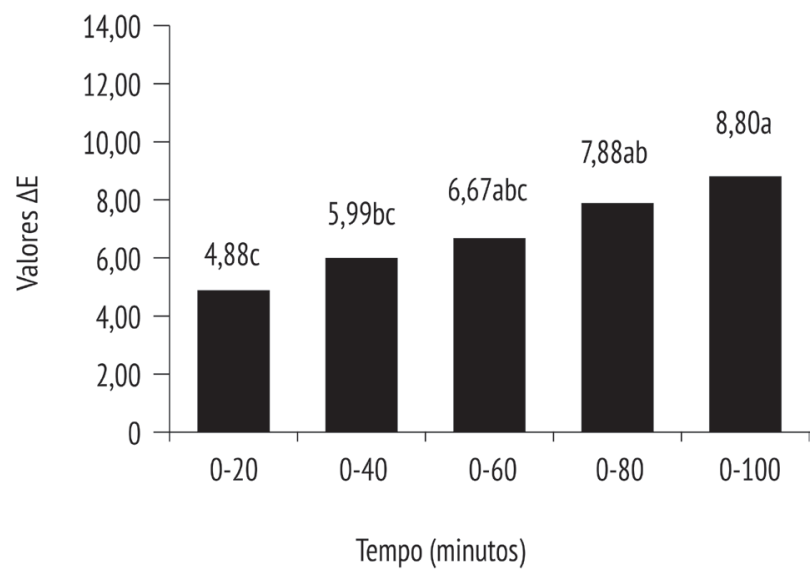

Figura 1 - Variações globais de cor $\left(\Delta \mathrm{E}_{0-20}, \Delta \mathrm{E}_{0-40}, \Delta \mathrm{E}_{0-60}, \Delta \mathrm{E}_{0-80}, \Delta \mathrm{E}_{0-100}\right)$ do músculo longissimus thoracis de bubalinos não maturado $(0$ dias de maturação) e maturado (14 dias de maturação) avaliado em diferentes tempos após abertura de embalagem a vácuo (0 dias de maturação: $P=0,001$ e $E P=0,399 ; 14$ dias de maturação: $P=0,001$ e $E P=0,284$ ). 
Além disso, os resultados demonstram que tanto as carnes maturadas quanto as não maturadas apresentaram mudança significativa nas variações globais da cor entre 0-100 minutos comparado ao tempo inicial $(0-20$ minutos $)(\mathrm{P}<0,05$; Figura 1), reforçando a falta de estabilidade até o último tempo avaliado (100 minutos).

É importante ressaltar que os valores médios de luminosidade e intensidade de vermelho encontram-se próximos aos reportados em carne de búfalos machos maturada $\left(2 \pm 1^{\circ} \mathrm{C}\right)$ por 0 e 15 dias, as quais apresentaram 33,35 e 32,39, 19,39 e 16,28 , para $L^{*}$ e a*, respectivamente (Irurueta et al., 2008). Por outro lado, a intensidade de amarelo encontra-se inferior aos valores encontrados por esses autores (15,68 e 12,91 para 0 e 15 dias de maturação, respectivamente). Entretanto, os valores médios encontrados para a cor da carne bubalina dos animais do presente estudo estão dentro dos padrões propostos por Muchenjea et al. (2009), os quais descrevem que as médias de luminosidade variam entre 33,2 - 41,0, as médias de a* entre 11,1 - 23,6 e as médias de b* entre 6,1 - 11,3.

Além disso, o pH 24 h após o abate $(5,69 \pm 0,1)$ apresentou-se dentro da faixa $(5,4$ a 6,0$)$ que garante os padrões normais de desenvolvimento das características físicas e químicas responsáveis pela qualidade da carne (Terlouw et al., 2008). Esse fato reforça que a falta de estabilização da cor da carne dessa espécie após 100 minutos da abertura da embalagem é condição intrínseca da carne, não estando relacionada a alterações do processo de transformação de músculo em carne (como DFD dry, firm and dark).

\section{Carne ovina}

Em relação à carne ovina, foi observado que aos 14 dias de maturação a intensidade de vermelho apresentou regressão quadrática $(\mathrm{P}<0,05)$ e para ambos os métodos de estocagem (não-maturada e maturada) houve o mesmo comportamento na intensidade de amarelo $(\mathrm{P}<0,05)$ (Tabela 2).

Tabela 2 - Cor instrumental do músculo biceps femoris de ovinos não maturado (0 dias de maturação) e maturado (14 dias de maturação), avaliado em diferentes tempos após ser retirado da embalagem a vácuo

\begin{tabular}{|c|c|c|c|c|c|c|c|c|}
\hline \multicolumn{9}{|c|}{0 dias de maturação } \\
\hline \multirow{2}{*}{ Itens } & \multicolumn{6}{|c|}{ Tempo de abertura da embalagem (min) } & \multirow{2}{*}{$\mathrm{CV}(\%)$} & \multirow{2}{*}{$\mathbf{R}$} \\
\hline & 0 & 20 & 40 & 60 & 80 & 100 & & \\
\hline $\mathrm{L}^{*}$ & 35,45 & 35,49 & 34,61 & 34,77 & 34,57 & 34,63 & 5,96 & Ns \\
\hline$a^{*}$ & 17,91 & 19,98 & 20,04 & 20,30 & 19,61 & 19,80 & 10,41 & Ns \\
\hline $\mathbf{b}^{*}$ & 5,97 & 9,45 & 9,67 & 10,14 & 10,02 & 10,34 & 14,34 & $Q^{1}$ \\
\hline \multicolumn{9}{|c|}{14 dias de maturação } \\
\hline \multirow{2}{*}{ Itens } & \multicolumn{6}{|c|}{ Tempo de abertura da embalagem (min) } & \multirow{2}{*}{$\mathrm{CV}(\%)$} & \multirow{2}{*}{$\mathbf{R}$} \\
\hline & 0 & 20 & 40 & 60 & 80 & 100 & & \\
\hline $\mathrm{L}^{*}$ & 38,15 & 39,51 & 38,94 & 38,00 & 37,95 & 37,07 & 8,91 & Ns \\
\hline$a^{*}$ & 19,02 & 22,32 & 22,88 & 22,82 & 22,70 & 22,72 & 7,62 & $Q^{1}$ \\
\hline $\mathbf{b}^{*}$ & 6,03 & 11,48 & 12,11 & 11,91 & 11,93 & 11,91 & 14,18 & $Q^{2}$ \\
\hline
\end{tabular}

Nota: $L^{*}=$ Luminosidade; $a^{*}=$ Intensidade de vermelho; $b^{*}=$ Intensidade de amarelo.

$\mathrm{CV}=$ Coeficiente de variação. $R=$ Regressão. Ns $=$ Não significativo. $Q=$ Regressão Quadrática.

0 dias de maturação: $Y 1=-0,0008 \times 2+0,1109 \mathrm{X}+6,525\left(R^{2}=0,87\right)$.

14 dias de maturação: $Y 1=-0,0008 \times 2+0,1105 x+19,561\left(R^{2}=0,85\right) \cdot Y 2=-0,0013 \times 2+0,1765 x+6,9422\left(R^{2}=0,83\right)$. 
Esse comportamento de regressão para a intensidade de vermelho e intensidade de amarelo nas carnes indica que diferentemente da carne bubalina, ocorre estabilidade da cor da carne ovina aos 69,31 minutos para $b^{*}$ em carne não maturada e 69,06 e 67,88 minutos para a* e b* em carne maturada por 14 dias, respectivamente, uma vez que a partir desse tempo os valores dessas coordenadas começam a reduzir.

De acordo com os resultados obtidos para os valores de $\Delta \mathrm{E}$ (Figura 2), não foi observada diferença entre os tempos 0 a 100 minutos $(\mathrm{P}>0,05)$. Embora os valores de $\Delta \mathrm{E}$ em cada período de tempo em relação a 0 minutos sejam considerados evidentes ao olho humano (Prändl et al., 1994), a ausência de diferença significativa entre os tempos avaliados reforça a estabilidade da cor da carne dessa espécie.

Quanto à cor da carne ovina comparada com a literatura, os valores de luminosidade e de intensidade de vermelho foram próximos aos encontrados em carne de ovelhas de descarte abatidas em distintos estágios fisiológicos de lactação (60 dias de lactação; 90 dias de lactação; e ovelhas não paridas no ano e que permaneceram por 60 dias junto às ovelhas dos grupos anteriores), os quais variaram de 37,56 a 41,13 e 17,92 a 18,79, respectivamente (Pinheiro et al., 2010). Valores próximos aos do presente estudo também foram encontrados em carne maturada de ovelhas de descarte durante zero, quatro e oito dias após 30 minutos de abertura da embalagem, sendo de $19,4,19,48$ e 20,41 para intensidade de vermelho e de 7,17, 8,38 e 8,54 para intensidade de amarelo, respectivamente para zero, quatro e oito dias de maturação a $5 \pm 2^{\circ} \mathrm{C}$ (Constantino et al., 2012).

Assim como a carne bubalina, a cor da carne ovina também apresentou variações naturais com o tempo de exposição à atmosfera que não estão relacionadas a alterações físico-químicas da carne, uma vez que o pH 24 h após o abate $(5,85 \pm 0,1)$ também se apresentou dentro da faixa $(5,4$ a 6,0$)$ que garante os padrões normais de desenvolvimento das características físicas e químicas responsáveis pela qualidade da carne (Terlouw et al., 2008).
0 dias de maturação

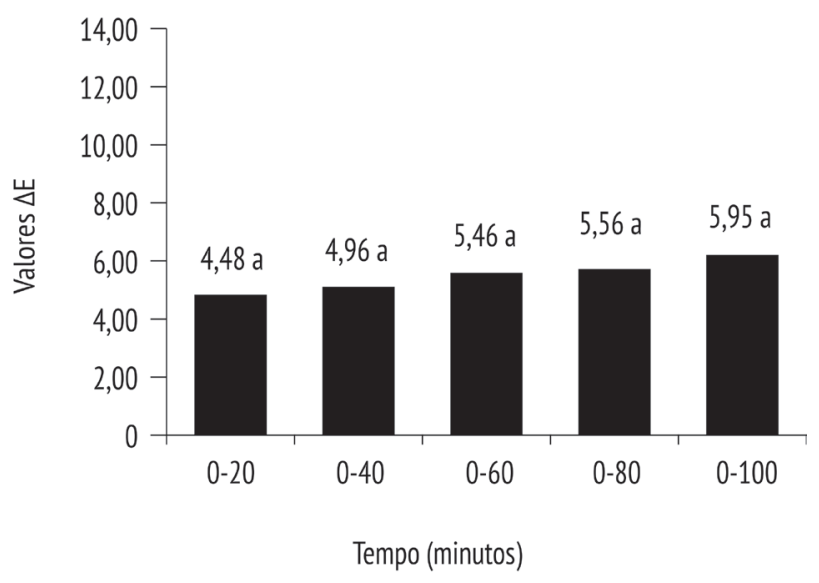

14 dias de maturação

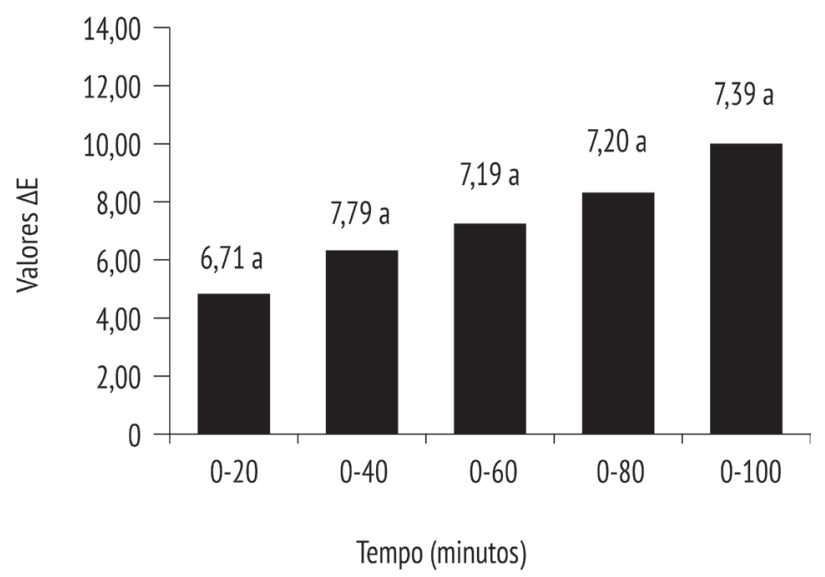

Figura 2 - Variações globais de cor $\left(\Delta \mathrm{E}_{0-20,}, \Delta \mathrm{E}_{0-40}, \Delta \mathrm{E}_{0-60}, \Delta \mathrm{E}_{0-80}, \Delta \mathrm{E}_{0-100}\right)$ do músculo Biceps femoris de ovinos não maturado (0 dias de maturação) e maturado (14 dias de maturação), avaliado em diferentes tempos após ser retirado da embalagem a vácuo (0 dias de maturação: $P=0,526$ e $E P=0,283 ; 14$ dias de maturação: $P=0,868$ e $E P=0,313$ ). 


\section{Conclusão}

Conclui-se que mesmo após 100 minutos de abertura da embalagem a vácuo da carne bubalina não-maturada e maturada $(0 \pm 1 \stackrel{\circ}{ } \mathrm{C}$ com ausência de luz) ainda ocorrem alterações na cor, sendo necessário rever para essa espécie a recomendação do tempo de abertura de 30 minutos da embalagem a vácuo para a estabilização da cor. Para a carne ovina não-maturada e maturada $\left(0 \pm 1^{\circ} \mathrm{C}\right.$ com ausência de luz), a estabilização da cor ocorre aos 67 e 69 minutos após a abertura da embalagem a vácuo. Assim, são necessários mais estudos para a carne dessas espécies quanto ao retorno da cor atrativa associada ao frescor, tanto para o conhecimento dos consumidores como para fins experimentais.

\section{Referências}

Abularach ML, Rocha CE, Felício PE. Características de qualidade de contrafilé ( $m$. L. dorsi) de touros jovens da raça Nelore. Cienc Tecnol Aliment. 1998;18(2):205-10.

AMSA - American Meat Science Association. Meat color measurement guidelines. Champaign, Illinois; 2012. $125 \mathrm{p}$.

Andrighetto C, Jorge AM, Roça RO, Sartori DR. Maturação da carne bovina. Rev Electron Vet. 2006;7(6):1-6.

Barducci RS, Franzói MCS, Sarti LMN, Millen DD, Putarov TC, Perdigão A, et al. Perfil de ácidos graxos e características da carne de bovinos nelore confinados com diferentes fontes lipídicas protegidas. Arq Bras Med Vet Zootec. 2016;68(1):233-42.

Barreto Neto AD. Posicionamento estratégico do setor de carnes de caprinos e ovinos no mercado de carnes brasileiro. Tecnol \& Cienc Agropec. 2010;4(4):81-5.

Constantino C, Ribeiro ELA, Bridi AM, Bridi AN, Tarsitano MA, Koritiaki NA, et al. Qualidade da carne maturada de ovelhas em sistema de embalagem a vácuo durante diferentes períodos de acondicionamento. Semin: Cienc Agrar. 2012;33(6Supl2):3437-46.

FAOSTAT - Food and Agriculture Organization of the
United Nations [acesso em 17 fev 2017] . Disponível em: http://faostat3.fao.org.

Giordano G, Guarini P, Ferrari P, Biondi-Zoccai G, Schiavone B, Giordano A. Beneficial impact on cardiovascular risk profile of water buffalo meat consumption. Eur J Clin Nutr. 2010;64(9):1000-6.

Giuffrida-Mendoza M, Moreno LA, Huerta-Leidenz N, Uzcátegui-Bracho S, Valero-Leala $\mathrm{K}$, Romero $\mathrm{S}$, et al. Cholesterol and fatty acid composition of longissimus thoracis from water buffalo (Bubalus bubalis) and Brahman-influenced cattle raised under savannah conditions. Meat Sci. 2015;106:44-9.

Honikel KO. Reference methods for the assessment of physical characteristics of meat. Meat Sci. 1998;49(4):447-57.

Huerta-Leidenz N, Rodas-González A, Vidal A, LopezNuñez J, Colina O. Carcass cut-out value and eating quality of longisssimus muscle from serially harvested savannahraised Brahman-influenced cattle and water buffaloes in Venezuela. Anim Prod Sci. 2016;56(12):2093-104.

Irurueta M, Cadoppi A, Langman L, Grigioni G, Carduza F. Effect of aging on the characteristics of meat from water buffalo grown the in the Delta del Paraná region of Argentina. Meat Sci. 2008;79(3):529-33.

Lawrie RA. Meat science. 4th ed. New York: Pergamon Press; 1985. 451 p.

Macdougal DB. Colour Meat. In: Pearson AM, Dutson TR (Eds.) Quality attributes and their measurement in meat, poultry and fish products - Advances in Meat Research Series. London: Blackie Academic \& Professional; 1994. 9 vol. p. 79-93.

Magalhães KA, Martins EC, Souza JDF, Barbosa CMP, Guimarães VP. Paranoma e perspectiva nacional da Ovinocultura e Caprinocultura. 2016 [acesso em $17 \mathrm{fev}$ 2017]. Disponível em: https://tinyurl.com/k6jjsor.

MAPA - Ministério da Agricultura, Pecuária e Abastecimento. Bovinos e Bubalinos [acesso em $17 \mathrm{fev}$ 2017]. Disponível em: http://www.agricultura.gov.br/ animal/especies/bovinos-e-bubalinos. 
Mello JLM, Rodrigues ABB, Giampietro-Ganeco A, Ferrari FB, Souza RA, Souza PA, et al. Characteristics of carcasses and meat from feedlot-finished buffalo and Bos indicus (Nellore) bulls. Anim Prod Sci. 2017. doi:10.1071/ AN16556.

Muchenjea V, Dzamac BK, Chimonyoa M, Stridom PE, Hugo A, Raats JG. Some biochemical aspects pertaining to beef eating quality and consumer health: a review. Food Chem. 2009;112:279-89.

Palka K. The influence of post-mortem ageing and roasting on the microestructure, texture and collagen solubility of bovine semitendinosus muscle. Meat Sci. 2003;64(2):191-8.

Pardi MC, Santos IF, Souza ER, Pardi HS. Ciência, higiene e tecnologia da carne. Goiânia: EDUFF/CEGRAF; 1995. $586 \mathrm{p}$.

Penny IF. Enzimologia de la maturation. In: Lawrie R (Ed.). Avances de la ciência de la carne. Zaragoza: Acribia; 1984. 148-81.

Pinheiro RSB, Jorge AM, Souza HBA, Boiago MM. Coloração da gordura e qualidade da carne de ovelhas de descarte abatidas em distintos estágios fisiológicos. Arq Bras Med Vet Zootec. 2010;62(2):468-74.

Prändl 0, Fischer A, Schmidhofer T, Sinell HJ. Tecnologia e higiene de la carne. Zaragoza: Acribia; 1994. 854 p.

Puga DMU, Contreras CJC, Turnbull MR. Avaliação do amaciamento de carne bovina de dianteiro (Triceps brachii) pelos métodos de maturação, estimulação elétrica, injeção de àcidos e tenderização mecânica. Cienc Tecnol Aliment. 1999;19(1):88-96.

SAEG - Sistema para Análises Estatísticas, Versão 9,1. Viçosa: Fundação Arthur Bernardes- UFV; 2007. Disponível em: www.ufv.br/saeg.

Santos ACR. Efeitos dos períodos de administração de cloridrato de zilpaterol e do tempo de confinamento no desempenho, características de carcaça e qualidade de carne de bovinos Nelore [dissertação]. Piracicaba: Universidade de São Paulo; 2015.
Terlouw EM, Arnould C, Auperin B, BerrI C, Le BihanDuval E, Deiss V, et al. Pre-slaughter conditions, animal stress and welfare: current status and possible future research. Animal. 2008;2(10):1501-17.

Viana JGA. Panorama geral da ovinocultura no mundo e no Brasil. Revista Ovinos. 2008;4(12).
Recebido em: 15/12/2016

Received in: 12/15/2016

Aprovado em: 13/04/2017

Approved in: 04/13/2017 